\title{
A RESULT OF BASS ON CYCLOTOMIC EXTENSION FIELDS ${ }^{1}$
}

JOHN H. SMITH

In [1] Bass stated the result given below as Proposition 1 and derived some consequences. His proof of the proposition itself, however, contains a gap; Lemmas 2 and 3 are false as stated. The purpose of this note is to fill the gap by proving the slightly stronger Proposition 2.

We retain the notation of [1]. In particular $k_{m}=k\left(\zeta_{m}\right)$ where $\zeta_{m}=e^{2 \pi i / m}$. The letters $m, n, a, b, c, d, r, s, t, u, v$ will denote nonnegative integers, $p$ is a prime integer, and $K=k(i)$.

Proposition 1. Given $k$ and $n$, there is an $m$ such that $k_{m}^{*^{m}} \cap k^{*} \subset k^{*^{n}}$.

Proposition 2. Given $k$ there is an $m$ such that for all $n, k_{m n}^{* n n} \cap k^{*} \subset k^{*^{n}}$.

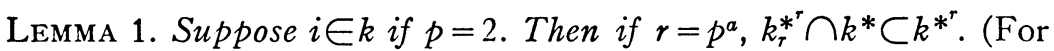
proof see p. 39 of [2].)

Lemma 2. Given $p$ and $k$ with $i \in k$ if $p=2$, suppose $r=p^{a}$ and $v$ are such that $\zeta_{p r} \notin k_{v}$. Then for all $t=p^{c}, k_{v}^{*^{r t}} \cap k^{*} \subset k^{*^{t}}$.

PROOF. If $c=0$ the result is trivial; assume $c>0$.

Case 1. $\zeta_{p} \in k$ or $\zeta_{p} \notin k_{v}$.

For any $u=p^{d}, d>0$, any $r u$ th power, $z \in k^{*}$ of an element in $k_{v}^{*}$ is a $p$ th power of an element in $k^{*}$. If not, $X^{r u}-z$ would be irreducible over $k$ [3, p. 221], hence all its roots would lie in $k_{v}$, which is normal over $k$, hence $\zeta_{r u} \in k_{v}$, contrary to supposition.

Therefore, if $x=y^{r t}, x \in k, y \in k_{v}^{*}$, then $x=w^{p}, w \in k^{*}$, and $w^{-1} y^{r t / p}$ is a $p$ th root of 1 in $k_{v}$, hence in $k$, and $y^{r t / p} \in k$. Repeating the argument if necessary we conclude, $y^{r} \in k^{*}, x=y^{r t} \in k^{*^{t}}$.

Case 2. $\zeta_{p} \notin k, \zeta_{p} \in k_{v}$.

If $x \in k$ is an $r t$ th power of something in $k_{v}$ then by Case $1, x$ is a $t$ th power of something in $k_{p} \subset k_{v}$. Taking norms from $k_{p}$ to $k$ and noting that $\left[k_{p}: k\right]$ is prime to $t$ gives the result.

Lemma 3. Let $s=2^{b}$ be such that $\zeta_{2 s} \notin K$. Then for any $t=2^{c}$, $K^{*^{* t}} \cap k^{*} \subset k^{*^{t}}$.

Received by the editors February 3, 1969.

1 Part of this work was done at a National Science Foundation Advanced Science Seminar at Bowdoin College, Summer, 1968. 
Proof (Following [2]). Let $x=y^{s t}, x \in k^{*}, y \in K^{*}$. If $y \in k^{*}$ there is nothing to prove, so assume $u=2^{d}$ such that $y^{u} \notin k^{*}, y^{2 u} \in k^{*}$. Then $y^{u}=i z, z \in k^{*}$ and if $\sigma$ denotes conjugation over $k,\left(y^{-1} y^{\sigma}\right)^{u}=-1$. Hence $u<s, y^{s} \in k^{*}, x=y^{s t} \in k^{*^{t}}$.

We are now ready to prove Proposition 2. For all ramified odd $p$ let $a_{p}$ denote one plus the exponent of $p$ in the ramification degree, from $Q$ to $k$, of some prime dividing $p$; for unramified odd $p$ let $a_{p}=0$, and let $a_{2}$ be one plus the exponent of 2 in the ramification degree, from $Q$ to $K$, of some prime dividing 2. Let $r_{p}=p^{a_{p}}$. Then for all $p$ and $v$ prime to $p, \zeta_{p r_{p}} \notin k_{v}$, in fact $\zeta_{2 r_{2}} \notin K_{v}$. Let $s_{p}=r_{p}$ for $p$ odd and $s_{2}=r_{2}^{2}$, and let $m=\Pi s_{p}$. Then for any $n=\Pi t_{p}, t_{p}=p^{c_{p}}$, letting $u_{p}=s_{p} t_{p}$,

$$
\begin{aligned}
k_{m n}^{*^{m n}} \cap k^{*} & =\left(\bigcap_{p} k_{m n}^{*^{u_{p}}}\right) \cap k^{*} \\
& \subset\left(\bigcap_{p \neq 2} k_{m n}^{*^{u_{p}}} \cap k_{m n / u_{p}}^{*}\right) \cap\left(K_{m n}^{*^{u_{2}}} \cap K_{m n / u_{2}}\right) \cap k^{*} \\
& \subset\left(\bigcap_{p \neq 2} k_{m n / u_{p}}^{*^{u_{p}}}\right) \cap\left(K_{m n / u_{2}}^{*_{u_{2}}}\right) \cap k^{*} \quad \text { (by Lemma 1) } \\
& \subset\left(\bigcap_{p \neq 2} k^{*^{t_{p}}}\right) \cap\left(K^{*^{r_{2} t_{2}}}\right) \cap k^{*} \quad \text { (by Lemma 2) } \\
& \subset \bigcap_{p}\left(k^{*^{t_{p}}}\right)=k^{*^{n}} \quad \text { (by Lemma 3). }
\end{aligned}
$$

Proposition 3. If $E=2 D_{k / Q}$, then $\bigcap_{r} k_{E^{r}} * E^{r}=\{1\}$.

Proof. $k$ contains no nontrivial roots of unity of order prime to $E$. Hence if $x \in k^{*}, x \neq 1, x \notin k^{*^{s}}$ for some $s=E^{b}$. The only odd primes in the $m$ of Proposition 2 are ramified ones, hence $m \mid t$ for some $t=E^{c}$. Then $x \notin k_{s t}^{* t} \cap k^{*} \subset k^{*^{s t / m}} \subset k^{*^{*}}$.

\section{REFERENCES}

1. H. Bass, A remark on an arithmetic theorem of Chevalley, Proc. Amer. Math. Soc. 16 (1965), 875-878. MR 32 \#2396.

2. C. Chevalley, Deux théorèmes d'arithmétique, J. Math. Soc. Japan 3 (1951), 36-44. MR 13, 440.

3. S. Lang, Algebra, Addison-Wesley, Reading, Mass., 1965. MR 33 \#5416.

Boston College 